\title{
Présentation de la traduction de John Searle (2006) : What is Language?
}

Guy Achard-Bayle

\section{(2) OpenEdition}

1 Journals

Édition électronique

URL : http://journals.openedition.org/pratiques/3519

DOI : 10.4000/pratiques.3519

ISSN : 2425-2042

Éditeur

Centre de recherche sur les médiations (CREM)

\section{Édition imprimée}

Date de publication : 15 décembre 2012

Pagination : 223-227

\section{Référence électronique}

Guy Achard-Bayle, "Présentation de la traduction de John Searle (2006) : What is Language? », Pratiques [En ligne], 155-156 | 2012, mis en ligne le 18 décembre 2017, consulté le 23 septembre 2020. URL : http://journals.openedition.org/pratiques/3519; DOI : https://doi.org/10.4000/pratiques.3519

(c) Tous droits réservés 


\section{Présentation de la traduction de John Searle (2006) : What is Language?}

John Searle nous fait l'honneur et l'amitié de nous laisser publier dans Pratiques la traduction en français de la conférence qu'il a donnée à Landau en 2006 : What is language?

John Searle était invité au $31^{\mathrm{e}}$ symposium LAUD ${ }^{(1)}$ organisé par l'université de Coblence-Landau en mars 2006. Comme on peut encore le voir sur le site de LAUD (http://www.linse.uni-due.de/linse/reportagen/LAUD_Symposium_2006.html), cette $31^{\mathrm{e}}$ édition du colloque était placée sous le thème de la pragmatique interculturelle, discipline ou notion alors toute récente ${ }^{(2)}$.

John Searle y donnait la conférence de clôture (qui comme on le verra dépasse largement la thématique citée) ; c'est à cette occasion qu'il nous a donné son accord pour la traduction; nous devions attendre toutefois que l'édition en anglais soit faite.

Nous présentons ici une version originale du texte, si l'on peut dire : cela signifie que d'autres versions ont vu le jour depuis, et l'on trouvera effectivement sur la page professionnelle de l'auteur (http://socrates.berkeley.edu/ jsearle/), le détail de ces publications : voir notamment, dans la rubrique Articles, une "expanded version" de notre texte, intitulée "What is Language: Some Preliminary Remarks", ou encore à la page 55 de sa Bibliography les références classées sous le $n^{\circ} 236^{(3)}$.

Mais pour ce qui nous concerne ici, c'est-à-dire la présentation de son texte traduit, nous voudrions signaler avant tout la parution en 2010 de son dernier ouvrage : Making the Social World. The Structure of Human Civilization (Oxford University Press).

Notre intérêt tout particulier pour cet ouvrage vient de ce que son chapitre 4 re-

(1) "Linguistic Agency University of Duisburg" : voir sur le site de LAUD 1'onglet "A Short History of LAUD”, http://www.uni-landau.de/anglistik/LAUD12/laud.htm

(2) Et largement « supportée » par la revue Intercultural Pragmatics crée en 2004 chez Mouton de Gruyter.

(3) Voir également (dans notre bibliographie) Searle 2007. 
prend et développe, autrement dit, prolonge le texte que nous traduisons ici. Les herméneutes de la pensée du philosophe auraient du grain à moudre, en étudiant les divers états du texte, son évolution ; mais pour nous, ce que nous retiendrons, en premier lieu, c'est que ce chapitre constitue l'aboutissement, donc l'un des cadres du texte que nous avons eu à traduire; et si nous n'allons pas, comme nous venons de le dire, nous lancer dans une génétique du texte, on peut observer, sans grand effort ou excès d'interprétation, que le titre des versions ultérieures, d'abord interrogatif, s'est fait assertif.

Nous voudrions souligner ensuite que le chapitre de l'ouvrage en question est intitulé "Language as Biological and Social" : c'est un excellent résumé de ce que nous allons lire, et un engagement fort en direction du "naturalisme " de l'auteur dont nous reparlerons plus bas, car c'est en effet une évolution récente et remarquable dans la pensée de l'auteur.

Enfin, on est avec ce chapitre 4 véritablement au cœur de la démonstration de l'ouvrage - il y a huit chapitres en tout; on peut donc aisément imaginer quelle place est faite au langage dans la somme que présente Searle dans cet ultime opus : le langage repose d'un côté sur l'intentionalité (Intentionality) ${ }^{(4)}$, individuelle et collective, et conditionne de l'autre la création des faits sociaux ou institutionnels.

Ainsi, Making the Social World fait très clairement le lien, et d'abord par son titre, avec un autre ouvrage majeur, The Construction of Social Reality $(1995)^{(5)}$ : nous le considérerons ici comme l'autre texte de référence, qui encadre « en amont » celui que nous traduisons et présentons. Mais comme nous allons le voir, les textes de Searle de la seconde moitié des années 2000 introduisent une notion, une dimension nouvelles, les développent, ce qui constitue une évolution capitale dans la pensée de l'auteur.

Car si l'on trouve bien à leur source la question sociale, les faits sociaux ou institutionnels, comme horizon de l'humanité, et donc comme définition de l'humain, les textes de Searle de cette fin de décennie mettent en avant sinon au jour deux éléments majeurs : la naturalisation du langage donc des faits institutionnels ou du social, et la déontologie ou l'engagement déontologique comme facteur constituant du langage, donc également du monde social.

Searle s'oriente ainsi vers un "philosophical project" (2010:3) de grande ampleur, dont la teneur est aussi anthropologique : "This book is concerned with the creation and maintenance of the distinctive features of human society and therefore, of human civilization" (ibid.) ; il donne à sa réflexion sur l'humain ou l'humanisation une dimension historique ou "évolutionniste », puisqu'il y est bien question d'apparition du langage et de développement d'aptitudes linguistiques comme conditions de l'évolution biologique, i.e. de l'apparition, des développements ou des perfectionnements de cet ensemble intentionnel et factuel qui fait proprement ou distinctement l'humanité.

L'autre nouveauté de ces textes est leur orientation «naturaliste » ${ }^{(6)}$. Searle a combattu plus d'une fois le relativisme constructiviste, pris position en faveur du réa-

(4) Suivant le titre de l'ouvrage de Searle (1983) et sa traduction en français par Claude Pichevin (1985).

(5) Traduit par Claudine Tiercelin (1998).

(6) Voir de premiers éléments en faveur de la naturalisation, in Searle (1992, trad. 1995 : 19-20). 
lisme (voir notamment les trois derniers chapitres, VII à IX, de Searle 1995); avec son engagement (et même son " parti pris » dès la première section de l'article à suivre) en faveur de la naturalisation du langage ("Naturalizing Language"), on peut dire qu'il enfonce le clou :

How is it possible in a universe consisting entirely of physical particles in fields of force that there can be such things as consciousness, intentionality, free will, language, society, ethics, aesthetics, and political obligations? (Searle, $2010: 3$ )

L'entreprise est donc aussi philosophique :

I have devoted most of my professional life to various aspects of it [la question posée précédemment]. This book uses my account of intentionality and my theory of speech acts to explain social ontology. (Ibid.)

Searle considère que notre monde ne fait qu'un, et son projet est d'expliquer comment on passe des électrons aux élections et des protons aux présidents. On voit ainsi se développer un schéma de dépendance qui va des faits bruts (1995) ou basiques (2010), relevant des sciences de la nature, aux faits institutionnels (1995) ou non basiques (2010), sociaux ou culturels, qui résultent de l'intentionalité et du langage ; les faits ou phénomènes de haut niveau, la vie sociale ou l'activité de l'esprit, dépendent de faits ou phénomènes de bas niveau, de la physique et de la biologie.

L'intentionalité quant à elle prend une forme collective pour donner naissance au social. On introduit alors l'aboutness, l'intentionalité étant par définition « à propos de »; autrement dit, elle caractérise les états mentaux qui sont «à propos de »-tous ne l'étant pas : l'anxiété, la nervosité par exemple... Donc pour les états dotés d'aboutness, l'intentionalité prend une forme ou mieux une structure propositionnelle, le type d'état $S$ et son contenu $p$ se représentant ainsi sous la forme : $S(p)$ - comme par ex. dans : Je crois, je crains, je désire (la pluie)... On se rapproche ainsi du langage.

À propos de langage, la conception naturaliste de Searle repose sur l'affirmation première que celui-ci est une extension de formes d'intentionalité prélinguistiques, que nous avons partagées avec les autres espèces animales ou d'hominidés (préhumains) au cours de l'évolution; à l'autre bout du processus évolutif d'hominisation, d'humanisation et de civilisation, le langage permet de créer le social : entre l'esprit et la société, le langage fait le pont ${ }^{(7)}$.

Autrement dit, si et quand il y a langage, il n'y a pas ou plus état de nature : si et quand existe un langage, il est (par définition) partagé, et exige donc (ou comme on le verra fait exister) un contrat social. Ainsi, si les hominidés ou les préhumains sont doués d'intentionalité, individuelle et collective, ils n' ont pas pour autant le langage - ou les langues ${ }^{(8)}$.

Reste à savoir ce que possède le langage, et non la conscience prélinguistique, et à

(7) "This [le chapitre 4 "Language as Biological and Social", in Searle 2010] is the bridge chapter between mind and society."

(8) Ceci dit, l'approche évolutionniste laisse attendre en premier lieu le langage : voir en la matière Bernard Victorri (2006) qui avec ses collègues recensent les hypothèses "paléolinguistiques » émises à partir des travaux de la génétique des populations. 
quel moment de l'évolution de l'esprit prélinguistique apparaît le langage ; autrement dit, pourquoi et comment on passe ainsi des hominidés aux humains.

L'intentionnalité prélinguistique et le langage, en acte, ont en commun, tout d'abord, une structure propositionnelle $S(p)$ qui détermine leurs conditions de satisfaction (et pour certains de vérité), ensuite une direction d'ajustement: soit dans le sens $\downarrow$ mot-monde (pour les assertions qui expriment des croyances, des perceptions), soit dans le sens $\uparrow$ monde-mot (pour les énoncés qui expriment des désirs, ou des ordres).

La conscience prélinguistique (comme celle de l'animal) possède également des catégories : espace, temps, identité, objet, etc. ; donc la faculté de distinguer ou discrétiser, reconnaître : identifier et réidentifier (le même individu), catégoriser (comme un même individu)...

Ce qui permet alors de différencier les deux stades de l'évolution, c'est que si de son côté la conscience prélinguistique se présente comme un flot continu, de son côté le langage n'existe comme tel qu'avec les éléments discrets qui le constituent et permettent de composer des énoncés où ces éléments discrets sont réutilisables, permutables, et même transformables.

Le langage se distingue enfin par ses actes déclaratifs ou performatifs (tels que promettre quelque chose) qui ont une double direction d'ajustement : l'acte de langage crée la réalité $\uparrow$ que l'énoncé représente $\downarrow$.

Nous arrivons maintenant à la deuxième question. L'apparition du langage en tant que tel est un passage à l'acte. Nous communiquons avant tout pour informer, mais si c'est pour informer de l'état du monde plus que de l'état de notre conscience, le passage à l'acte consiste bien en la reproduction du contenu propositionnel d'un état mental, ou intentionnel. Searle envisage ainsi, comme d'autres (voir bibliographie), un stade de développement protolinguistique, "sans syntaxe », fait par exemple de propositions exclamatives réduites à un seul lexème, et censées informer mais pour agir, avertir, prévenir : Feu ! Pluie ! Ceci conduit au «langage réel », avec ses trois caractéristiques : discrétion, compositionnalité, générativité ${ }^{(9)}$.

Les « paléolinguistes » s'accordent à dire, comme Searle, que l'information expression de la vérité conduit vite à sa défense. Ainsi, quand un locuteur produit un énoncé, il veut que cet énoncé réussisse : pour cela, il faut des outils conventionnels, i.e. reconnus socialement; dès lors, il ne suffit plus d'exprimer des états, de faits et intentionnels, par de courtes exclamations comme comme celles citées supra, il faut faire des énoncés, et faire de leurs éléments des représentations.

La dernière étape dans le modèle searlien ancré au biologique débouche sur la création de la réalité sociale, en faisant intervenir la déontologie : cela se passe ou s'accomplit dès lors que le locuteur non seulement s'assure de... mais également s'engage sur la vérité de ce qu'il dit; de plus, il s'engage explicitement et publiquement : ainsi une assertion engage le locuteur sur la vérité de son contenu, une promesse l'engage à la tenir; mais dans les deux cas, l'engagement fait partie de l'acte de langage qui s'accomplit : c'est un acte public où le locuteur engage sa parole.

L'ultime degré de socialisation est franchi par extension de ce principe déontologique : quand on dit, par exemple, «C'est notre chef», «C'est ma maison », on ne fait

(9) Voir là encore des exemples in Dessalles, Picq \& Victorri (2006). 
pas que représenter des états de choses, on les crée, et on les crée avec une nouvelle déontologie dans la mesure où les autres en conviennent.

Nous utilisons donc le langage pour rendre compte des phénomènes et des faits, mais aussi créer ceux qui constitueront ainsi la réalité institutionnelle : la réalité du gouvernement, de la propriété privée, du mariage, comme celle de la monnaie, des universités ou des cocktails, sont essentiellement linguistiques. En un mot : le langage ne fait pas que décrire, il crée, et en partie constitue, ce qu'il décrit.

\author{
Guy Achard-Bayle ${ }^{(10)}$ \\ Université de Lorraine, \\ CREM-Praxitexte
}

\title{
Références
}

Dessalles, Jean-Louis, PICQ, Pascal \& ViCTORRI, Bernard (2006, 2010²), Les origines du langage, Paris, Le Pommier.

VICTORRI, Bernard $\left(2006,2010^{2}\right)$, « À la recherche de la langue originelle », in Jean-Louis Dessalles et al., 77-124.

SEARLE John :

(1969), Speech Acts, trad. Hélène Pauchard (1972), Les actes de langage. Essai de philosophie du langage, Paris, Hermann.

(1983), Intentionality. An essay in philosophy of mind, trad. Claude Pichevin (1985), L'Intentionalité. Essai de philosophie des états mentaux, Paris, Éditions de Minuit.

(1992), The Rediscovery of Mind, trad. Claudine Tiercelin (1995), La redécouverte de l'esprit, Paris, Gallimard.

(1995), The Construction of Social Reality, trad. Claudine Tiercelin (1998), La construction de la réalité sociale, Paris, Gallimard.

(2006), What is language?, Conférence au $31^{\mathrm{e}}$ symposium LAUD Landau (traduction ci-dessous).

(2007), What is language: Some preliminary remarks, in Explorations in Pragmatics: Linguistic, Cognitive, and Intercultural Aspects, Istvan Kecskes \& Laurence Horn (eds), Berlin-New York, Mouton de Gruyter, 7-38.

(2010), Making the Social World. The Structure of Human Civilization, Oxford, Oxford University Press.

(10) Je dois et je dis un grand merci aux collègues anglicistes et linguistes de l'Université de Lorraine qui m'ont aidé à la traduction et à la relecture du texte traduit : Kathie Birat, Yvon Keromnes et Caroline Masseron. 